\title{
A New Method for Ultrashort Electron Pulse-shape Measurement Using Synchrotron Radiation from a Bending Magnet
}

\author{
J. Krzywinski $^{\text {a }}$, E.L. Saldin ${ }^{\text {b }}$, E.A. Schneidmiller ${ }^{\text {b }}$, M.V. Yurkov ${ }^{c}$ \\ ${ }^{a}$ Institute of Physics of the Polish Academy of Sciences, 02688 Warszawa, Poland \\ b Automatic Systems Corporation, 443050 Samara, Russia \\ c Joint Institute for Nuclear Research, Dubna, 141980 Moscow Region, Russia
}

\begin{abstract}
A new non-destructive method for measurement of the longitudinal profile of subpicosecond electron bunches is proposed. The method is based on measurement of spectral intensity correlations in synchrotron radiation. Statistical properties of synchrotron radiation produced by a bunch passing the bending magnet are considered. The signal to noise ratio is analyzed in terms of the degeneracy parameter. The degeneracy parameter increases approximately as a third power of the wavelength which makes the visible range of synchrotron radiation to be a natural choice for the spectral intensity correlation measurement. An example of the experimental set-up is also described.
\end{abstract}




\section{Introduction}

The length of electron bunches for the next generation linear colliders are of an order of $\sigma_{z} \simeq 0.1-1 \mathrm{~mm}[1-3]$. The projects of X-ray FELs require even shorter bunches, down to $0.025 \mathrm{~mm}[1,4,5]$. These values are less by an order of magnitude than those used in the existent accelerators. Development of nondestructive methods for measurements of longitudinal distribution of the beam current in such a short bunches is a challenging problem.

In this paper we describe a new method for nondestructive measurement of the longitudinal profile of the electron bunch. The method is based on the measurements in frequency domain of correlations between the fluctuations of synchrotron radiation produced by a bunch passing a bending magnet.

The proposed device is the combination of a monochromator and a counting interferometer which gives directly the square of the modulus of the Fourier transform of the longitudinal electron bunch profile. Reconstruction of the beam profile from these data is performed by means of a standard technique developed for the image reconstruction from the data obtained by means of the Hanbury-Brown and Twiss intensity interferometer.

This paper is organized as follows. The principle of the method is described in section 2. The principle of operation of the proposed device is based essentially on the statistical properties of the synchrotron radiation and the detection process itself, so the next five sections are devoted to detailed description of these topics. Statistical properties of synchrotron radiation are studied in section 3 . Section 4 is devoted to the problem of photoelectric detection of synchrotron radiation. Section 5 presents practical formulae for calculations of the spectral brightness of SR sources, of the wave degeneracy parameter and of the photocount degeneracy parameter. Sections 6 and 7 present a detailed study of the output characteristics of the device. In the end of the paper we illustrate with numerical example the potential of the proposed method for an on line, non-destructive diagnostic of the electron beam in the accelerator at the TESLA Test Facility at DESY.

\section{Principle of the method}

The layout of the device for measurement the longitudinal profile of the electron pulse is presented in Fig. 1. An ultrarelativistic electron bunch passes a bending magnet and radiates a pulse of synchrotron radiation. The diaphragm of aperture $d$ is used for selection of the transversely coherent fraction of synchrotron radiation which is 


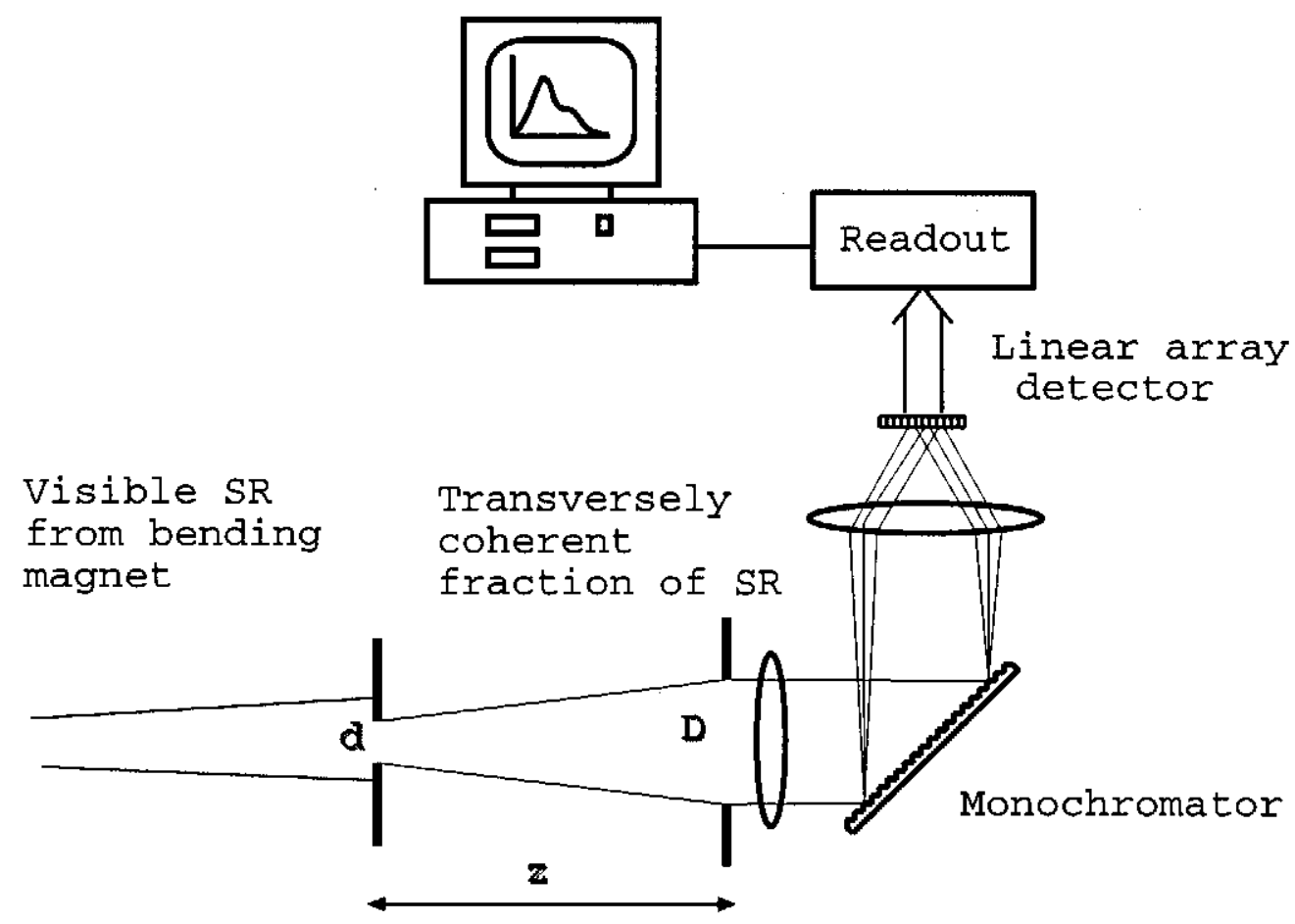

Fig. 1. The layout of the device for measurement the longitudinal profile of the electron pulse.

directed to the monochromator with an aperture $D$. The monochromator is placed at the distance of $z$ from the diaphragm. The resolution of the monochromator is equal to $\Delta \omega_{m}$, the central frequency is equal to $\omega_{0}$ and $\Delta \omega_{m} / \omega_{0} \ll 1$. The radiation reaching the monochromator is transversely coherent when, according to Van Cittert-Zernike theorem, the following condition is fulfilled (see e.g. [6]):

$$
\frac{c z}{\omega_{0} d}>D
$$

where $c$ is the velocity of light. A one-dimensional array photodetector is placed at the monochromator exit which performs measurement-of the spectral distribution of the radiation energy. The signal from each element of the photodetector is stored in the computer in matrix form. The row index corresponds to the frequency and the column index corresponds to the pulse number. This matrix is used for the calculation of statistical properties of the radiation. In particular, the spectral correlation function contains the information about the Fourier transform of the electron bunch profile.

The principle of operation of the proposed device is based essentially on the statistical properties of synchrotron radiation. It is shown in section 2 that synchrotron radiation possesses all the features corresponding to completely chaotic polarized light. In particular, the higher order spectral correlation functions are expressed via the first 
order correlation function. The probability density distribution of the spectral density of the radiation bunch energy follows the negative exponential distribution and the probability density function of the radiation bunch energy after monochromator follows the gamma distribution.

At sufficient resolution of the monochromator, it seems to be technically feasible to measure the second order spectral correlation function. The results of spectral correlation measurements can be used for the reconstruction of the electron pulse shape.

When resolution of the monochromator is worse than the interval of spectral coherence, $\Delta \omega_{c} \simeq c / \sigma_{z}$, the mean value-to-dispersion ratio of the photocounts in a given frequency range $\Delta \omega_{m}$ can be measured. The square of this ratio is equal to $M$, the number of coherence intervals inside the monochromator linewidth. Analysis of these measurements allows one to estimate the electron bunch length as $\sigma_{z} \simeq c M / \Delta \omega_{m}$.

For this method to be applicable the radiation wavelength must be much smaller than the bunch length. At a bunch length of the order of $0.1 \mathrm{~mm}$ one can use a wide interval of the radiation spectrum, from the infrared down to X-rays. The choice of the optimal value of the operating frequency is influenced by such issues as tolerable value of the signal-to-noise ratio, required resolution of the monochromator $\left(\Delta \omega_{m} / \omega \ll c /\left(\omega \sigma_{z}\right)\right)$ and the existence of commercially available detectors and optical elements with the required parameters. The choice of the visible range possesses the following advantages. The degeneracy parameter (number of photocounts in the interval of spectral coherence $\Delta \omega_{c}$ ) can reach a value larger than unity and therefore can ensure short measurement time. The required resolution of the monochromator $\left(\Delta \omega_{m} / \omega \ll c /\left(\omega \sigma_{z}\right) \simeq 0.1 \%\right)$ can be achieved without significant efforts. There is also a highly developed technology of electrooptical devices (optical fibers, fast detectors, electrooptical switches, etc.) operating in the visible wavelength range.

\section{Statistical properties of synchrotron radiation}

Synchrotron radiation is a stochastic object and at a given time it is impossible to predict the amount energy which flows to a detector. The stochastic nature of the synchrotron radiation is determined by the shot noise of the electron bearn generating the radiation. 


\subsection{Shot noise in the electron beam}

Let us consider microscopic picture of the electron beam current at the entrance into a bending magnet. The electron beam current is constituted by moving electrons randomly arriving to the entrance of the magnet:

$$
I(t)=(-e) \sum_{k=1}^{N} \delta\left(t-t_{k}\right)
$$

where $\delta(\ldots)$ is the delta-function, (-e) is the charge of the electron, $N$ is the number of electrons in a bunch and $t_{k}$ is the arrival time of the $k$ th electron to the magnet entrance. The electron bunch profile is described by the profile function $F(t)$ and the beam current averaged over an ensemble of bunches can be written in the form:

$$
\langle I(t)\rangle=(-e) N F(t)
$$

The probability of the arrival of the electron at the time interval $(t, t+d t)$ is equal to $F(t) d t$. For the Gaussian profile the electron bunch function $F(t)$ has the form:

$$
F(t)=\frac{1}{\sqrt{2 \pi} \sigma_{T}} \exp \left(-\frac{t^{2}}{2 \sigma_{T}^{2}}\right)
$$

The electron beam current $I(t)$ and its Fourier transform $\bar{I}(\omega)$ are connected by Fourier transformations:

$$
\begin{aligned}
& \bar{I}(\omega)=\int_{-\infty}^{\infty} e^{i \omega t} I(t) d t=(-e) \sum_{k=1}^{N} e^{i \omega t_{k}} \\
& I(t)=\frac{1}{2 \pi} \int_{-\infty}^{\infty} \bar{I}(\omega) e^{-i \omega t} d \omega=(-e) \sum_{k=1}^{N} \delta\left(t-t_{k}\right)
\end{aligned}
$$

It follows from eq. (3) that Fourier transform of the input current, $\bar{I}(\omega)$, is the sum of a large number of complex random phasors with random phases $\phi_{k}=\omega t_{k}$. If the characteristic duration of a bunch $\sigma_{T}$ is long, $\omega \sigma_{T} \gg 1$, then the phases $\phi_{k}$ can be considered to be uniformly distributed on interval $(\pi,-\pi)$. Under this condition the probability density distribution of $|\bar{I}(\omega)|^{2}$ is given by the negative exponential distribution [6]:

$$
p\left(|\bar{I}(\omega)|^{2}\right)=\frac{1}{\left\langle|\bar{I}(\omega)|^{2}\right\rangle} \exp \left(-\frac{|\bar{I}(\omega)|^{2}}{\left\langle|\bar{I}(\omega)|^{2}\right\rangle}\right)
$$


where $\langle\ldots\rangle$ means the averaging over an ensemble of bunches.

Let us calculate the first order correlation of complex Fourier harmonics $\bar{I}(\omega)$ and $\bar{I}\left(\omega^{\prime}\right)$ :

$$
\left\langle\bar{I}(\omega) \bar{I}^{*}\left(\omega^{\prime}\right)\right\rangle=e^{2}\left\langle\sum_{k=1}^{N} \sum_{n=1}^{N} \exp \left(i \omega t_{k}-i \omega^{\prime} t_{n}\right)\right\rangle .
$$

Expanding this relation, we can write:

$$
\begin{array}{r}
\left\langle\bar{I}(\omega) \bar{I}^{*}\left(\omega^{\prime}\right)\right\rangle=e^{2}\left\langle\sum_{k=1}^{N} \exp \left[i\left(\omega-\omega^{\prime}\right) t_{k}\right]\right\rangle+e^{2}\left\langle\sum_{k \neq n} \exp \left(i \omega t_{k}-i \omega^{\prime} t_{n}\right)\right\rangle \\
=e^{2} \sum_{k=1}^{N}\left\langle\exp \left[i\left(\omega-\omega^{\prime}\right) t_{k}\right]\right\rangle+e^{2} \sum_{k \neq n}\left\langle\exp \left(i \omega t_{k}\right)\right\rangle\left\langle\exp \left(-i \omega^{\prime} t_{n}\right)\right\rangle .
\end{array}
$$

Taking into account the relation (1) and the first relation in the eq. (3) we obtain that $\left\langle\exp \left(i \omega t_{k}\right)\right\rangle$ is equal to the Fourier transformation of the bunch profile function $F(t)$ :

$$
\left\langle\exp \left(i \omega t_{k}\right)\right\rangle=\int_{-\infty}^{\infty} F\left(t_{k}\right) e^{i \omega t_{k}} d t_{k}=\bar{F}(\omega),
$$

Fourier transform of the Gaussian profile function (2) has the form:

$$
\bar{F}(\omega)=\exp \left(-\frac{\omega^{2} \sigma_{T}^{2}}{2}\right)
$$

Substituting (5) in the expression (4) we obtain:

$$
\left\langle\bar{I}(\omega) \bar{I}^{*}\left(\omega^{\prime}\right)\right\rangle=e^{2} N \bar{F}\left(\omega-\omega^{\prime}\right)+e^{2} N(N-1) \bar{F}(\omega) \bar{F}^{*}\left(\omega^{\prime}\right)
$$

In the case when

$$
N|\bar{F}(\omega)|^{2} \ll 1
$$

the expression for the first order spectral correlation takes the form:

$$
\left\langle\bar{I}(\omega) \bar{I}^{*}\left(\omega^{\prime}\right)\right\rangle=e^{2} N \bar{F}\left(\omega-\omega^{\prime}\right) \text {. }
$$


For the case of the electron bunch with the Gaussian profile, the explicit expression for the first order correlation of complex Fourier harmonics $\bar{I}(\omega)$ and $\bar{I}\left(\omega^{\prime}\right)$ has the form:

$$
\left\langle\bar{I}(\omega) \bar{I}^{*}\left(\omega^{\prime}\right)\right\rangle=e^{2} N \exp \left[-\frac{\left(\omega-\omega^{\prime}\right)^{2} \sigma_{T}^{2}}{2}\right] .
$$

Let us discuss the applicability region of approximation (6). The physical sense of relation (6) is that frequency $\omega$ has to be large enough. Let us consider numerical example for the case of the Gaussian profile of the electron bunch. At $\omega \sigma_{T}=10$ we have $|\bar{F}(\omega)|^{2}=\exp (-100) \simeq 4 \times 10^{-44}$. As a rule, the number of particles in the bunch $N$ is not larger than $10^{11}$, so condition (6) is fulfilled in practice.

Let us calculate the second order correlation of complex Fourier harmonics $\bar{I}(\omega)$ and $\bar{I}\left(\omega^{\prime}\right)$ :

$$
\left\langle|\bar{I}(\omega)|^{2}\left|\bar{I}\left(\omega^{\prime}\right)\right|^{2}\right\rangle=e^{4}\left\langle\sum_{n=1}^{N} \sum_{m=1}^{N} \sum_{p=1}^{N} \sum_{q=1}^{N} \exp \left[i \omega\left(t_{n}-t_{m}\right)+i \omega^{\prime}\left(t_{p}-t_{q}\right)\right]\right\rangle .
$$

The $N^{4}$ terms in this summation can be placed in 15 different classes (see, e.g. ref. [6]). When condition (6) is fulfilled, only two classes are of importance with ( $n=m, p=q$, $n \neq p)$ and $(n=q, m=p, n \neq m)$. Thus, we can write:

$$
\left\langle|\bar{I}(\omega)|^{2}\left|\bar{I}\left(\omega^{\prime}\right)\right|^{2}\right\rangle=\left\langle|\bar{I}(\omega)|^{2}\right\rangle\left\langle\left|\bar{I}\left(\omega^{\prime}\right)\right|^{2}\right\rangle+\left|\left\langle\bar{I}(\omega) \bar{I}^{*}\left(\omega^{\prime}\right)\right\rangle\right|^{2} .
$$

Substituting eq. (7) into eq. (9) we obtain:

$$
\left\langle|\bar{I}(\omega)|^{2}\left|\bar{I}\left(\omega^{\prime}\right)\right|^{2}\right\rangle=e^{4} N^{2}\left(1+\left|\bar{F}\left(\omega-\omega^{\prime}\right)\right|^{2}\right)
$$

\subsection{Analysis of synchrotron radiation properties in frequency domain}

Let us study the spectral characteristics of the transversely coherent fraction of synchrotron radiation after the monochromator. For simplicity we consider linear polarization of synchrotron radiation, and use scalar representation of the radiation field. Nevertheless, all the results are valid for any polarization. The central frequency of the monochromator is equal to $\omega_{0}$ and the bandwidth of monochromator is small. The linearity of Maxwell equations and the fact that a monochromator can be treated as a linear filter allows one to write the Fourier components of electric field of the synchrotron radiation in the following form:

$$
\bar{E}(\omega)=A(\omega) G_{m}\left(\omega-\omega_{0}\right) \bar{I}(\omega)
$$


where $G_{m}\left(\omega-\omega_{0}\right)$ is the frequency profile of the monochromator and $A(\omega)$ is the Fourier harmonic of the radiation field emitted by single electron. Taking into account that the linewidth of monochromator is small, we assume $A(\omega)$ to be constant within the monochromator bandwidth.

It follows from eq. (11) that statistical properties of the Fourier amplitudes $\bar{E}(\omega)$ are defined by statistical properties of the Fourier amplitudes of the input current $\bar{I}(\omega)$. In particular, it follows immediately from (3) that $|\vec{E}(\omega)|^{2}$ is distributed in accordance with the negative exponential probability density function:

$$
p\left(|\bar{E}(\omega)|^{2}\right)=\frac{1}{\left\langle|\bar{E}(\omega)|^{2}\right\rangle} \exp \left(-\frac{|\bar{E}(\omega)|^{2}}{\left\langle|\bar{E}(\omega)|^{2}\right\rangle}\right) .
$$

It should be noted that such a distribution is the feature of completely chaotic polarized radiation (see ref. [6] for more details).

Let us calculate the correlation of complex Fourier harmonics $\bar{E}(\omega)$ and $\bar{E}\left(\omega^{\prime}\right)$ :

$$
\left\langle\bar{E}(\omega) \bar{E}^{*}\left(\omega^{\prime}\right)\right\rangle=\left|A\left(\omega_{0}\right)\right|^{2} G_{m}\left(\omega-\omega_{0}\right) G^{*}\left(\omega^{\prime}-\omega_{0}\right)\left\langle\bar{I}(\omega) \bar{I}^{*}\left(\omega^{\prime}\right)\right\rangle .
$$

Substituting eq. (7) into eq. (13) we obtain:

$$
\left\langle\bar{E}(\omega) \bar{E}^{*}\left(\omega^{\prime}\right)\right\rangle=e^{2} N\left|A\left(\omega_{0}\right)\right|^{2} G_{m}\left(\omega-\omega_{0}\right) G_{m}^{*}\left(\omega^{\prime}-\omega_{0}\right) \bar{F}\left(\omega-\omega^{\prime}\right) .
$$

The average spectral density of the radiation energy at the detector installed after the monochromator is given by the expression:

$$
\frac{c}{4 \pi^{2}}\left\langle|\bar{E}(\omega)|^{2}\right\rangle=\frac{c e^{2} N}{4 \pi^{2}}\left|A\left(\omega_{0}\right)\right|^{2}\left|G_{m}\left(\omega-\omega_{0}\right)\right|^{2}
$$

The first order spectral correlation function is defined as

$$
g_{1}\left(\omega-\omega^{\prime}\right)=\frac{\left\langle\bar{E}(\omega) \bar{E}^{*}\left(\omega^{\prime}\right)\right\rangle}{\sqrt{\left\langle|\bar{E}(\omega)|^{2}\right\rangle\left\langle\left|\bar{E}\left(\omega^{\prime}\right)\right|^{2}\right\rangle}} .
$$

Substituting eqs. (14) and (15) into eq. (16) we obtain

$$
g_{1}\left(\omega-\omega^{\prime}\right)=\bar{F}\left(\omega-\omega^{\prime}\right)
$$

Explicit expression for the first order spectral correlation function of the SR emitted by the electron bunch with Gaussian bunch has the form:

$$
g_{1}\left(\omega-\omega^{\prime}\right)=\exp \left[-\frac{\left(\omega-\omega^{\prime}\right)^{2} \sigma_{T}^{2}}{2}\right] \text {. }
$$


We define the interval of spectral coherence $\Delta \omega_{c}$ as follows:

$$
\Delta \omega_{c}=\int_{-\infty}^{\infty}\left|g_{1}(\Delta \omega)\right|^{2} d(\Delta \omega)
$$

For the case of the Gaussian profile of the electron beam, the interval of spectral coherence is equal to

$$
\Delta \omega_{c}=\int_{-\infty}^{\infty}|\bar{F}(\Delta \omega)|^{2} d(\Delta \omega)=\frac{\sqrt{\pi}}{\sigma_{T}}
$$

The second order correlation of complex Fourier harmonics $\bar{E}(\omega)$ and $\bar{E}\left(\omega^{\prime}\right)$ is given by the expression:

$$
\begin{aligned}
\left\langle|\bar{E}(\omega)|^{2}\left|\bar{E}\left(\omega^{\prime}\right)\right|^{2}\right\rangle= & \left|A\left(\omega_{0}\right)\right|^{4}\left|G_{m}\left(\omega-\omega_{0}\right)\right|^{2}\left|G\left(\omega^{\prime}-\omega_{0}\right)\right|^{2} \\
& \times\left\langle|\bar{I}(\omega)|^{2}\left|\bar{I}\left(\omega^{\prime}\right)\right|^{2}\right\rangle .
\end{aligned}
$$

Using eqs. (9) and (13) we obtain:

$$
\left\langle|\bar{E}(\omega)|^{2}\left|\bar{E}\left(\omega^{\prime}\right)\right|^{2}\right\rangle=\left\langle|\bar{E}(\omega)|^{2}\right\rangle\left\langle\left|\bar{E}\left(\omega^{\prime}\right)\right|^{2}\right\rangle+\left|\left\langle\bar{E}(\omega) \bar{E}^{*}\left(\omega^{\prime}\right)\right\rangle\right|^{2}
$$

The second order spectral correlation function is defined as

$$
g_{2}\left(\omega-\omega^{\prime}\right)=\frac{\left\langle|\bar{E}(\omega)|^{2}\left|\tilde{E}\left(\omega^{\prime}\right)\right|^{2}\right\rangle}{\left\langle|\bar{E}(\omega)|^{2}\right\rangle\left\langle\left|\bar{E}\left(\omega^{\prime}\right)\right|^{2}\right\rangle}
$$

Using eqs. (22), (17) and (23) we obtain that the first and the second order correlation functions are connected by the relation:

$$
g_{2}\left(\omega-\omega^{\prime}\right)=1+\left|g_{1}\left(\omega-\omega^{\prime}\right)\right|^{2}
$$

which is also a general property of the completely chaotic polarized radiation. Explicit expression for the second order spectral correlation function has the form:

$$
g_{2}\left(\omega-\omega^{\prime}\right)=1+\left|\bar{F}\left(\omega-\omega^{\prime}\right)\right|^{2} .
$$

The next problem is a description of the fluctuations of the energy of the radiation pulse $W$ at the detector installed after the monochromator. From the expression for 
Pointing's vector and Parseval's theorem we have:

$$
W=\frac{c S}{4 \pi^{2}} \int_{0}^{\infty}|\bar{E}(\omega)|^{2} d \omega
$$

where $S$ is the transverse area of the detector. Taking into account eq. (15) we can write the expression for the average energy measured by the detector:

$$
\left.\langle W\rangle=\frac{c S}{4 \pi^{2}} \int_{0}^{\infty}\left\langle|\bar{E}(\omega)|^{2}\right\rangle d \omega=\frac{c e^{2} N S}{4 \pi^{2}}\left|A\left(\omega_{0}\right)\right|^{2} \int_{0}^{\infty} \mid G_{m}\left(\omega-\omega_{0}\right)\right)\left.\right|^{2} d \omega .
$$

One can obtain from this expression that the average energy is function of the frequency profile of the monochromator.

The normalized dispersion of the energy distribution is calculated as follows:

$$
\begin{aligned}
\sigma_{W}^{2}= & \frac{\left\langle(W-\langle W\rangle)^{2}\right\rangle}{\langle W\rangle^{2}} \\
= & \frac{\int_{0}^{\infty} d \omega \int_{0}^{\infty} d \omega^{\prime}\left\langle|\bar{E}(\omega)|^{2}\left|\bar{E}\left(\omega^{\prime}\right)\right|^{2}\right\rangle}{\int_{0}^{\infty} d \omega\left\langle|\bar{E}(\omega)|^{2}\right\rangle \int_{0}^{\infty} d \omega^{\prime}\left\langle\left|\bar{E}\left(\omega^{\prime}\right)\right|^{2}\right\rangle}-1
\end{aligned}
$$

Using definition (23) for the second order correlation function and relation (24) we reduce this expression to the form

$$
\frac{\left\langle(W-\langle W\rangle)^{2}\right\rangle}{\langle W\rangle^{2}}=\frac{\int_{0}^{\infty} d \omega \int_{0}^{\infty} d \omega^{\prime}\left\langle|\bar{E}(\omega)|^{2}\right\rangle\left\langle\left|\bar{E}\left(\omega^{\prime}\right)\right|^{2}\right\rangle\left|g_{1}\left(\omega-\omega^{\prime}\right)\right|^{2}}{\int_{0}^{\infty} d \omega\left\langle|\bar{E}(\omega)|^{2}\right\rangle \int_{0}^{\infty} d \omega^{\prime}\left\langle\left|\bar{E}\left(\omega^{\prime}\right)\right|^{2}\right\rangle}
$$

Analysis of this expression shows that the energy deviation after the monochromator depends on the frequency profile of the monochromator and on the electron bunch formfactor $F(\Delta \omega)$.

Let us consider the case of the electron bunch with the Gaussian profile and the monochromator with a rectangular line:

$$
\begin{aligned}
& \left|G_{m}\left(\omega-\omega_{0}\right)\right|^{2}=1 \quad \text { at }\left|\omega-\omega_{0}\right|<\frac{\Delta \omega_{m}}{2} \\
& \left|G_{m}\left(\omega-\omega_{0}\right)\right|^{2}=0 \text { at }\left|\omega-\omega_{0}\right|>\frac{\Delta \omega_{m}}{2} .
\end{aligned}
$$


Integration of expression (28) provides the following result:

$$
\sigma_{W}^{2}=\frac{\Delta \omega_{c}}{\Delta \omega_{m}} \operatorname{erf}\left(\sqrt{\pi} \frac{\Delta \omega_{m}}{\Delta \omega_{c}}\right)-\frac{1}{\pi}\left(\frac{\Delta \omega_{c}}{\Delta \omega_{m}}\right)^{2}\left\{1-\exp \left[-\pi\left(\frac{\Delta \omega_{m}}{\Delta \omega_{c}}\right)^{2}\right]\right\}
$$

where $\operatorname{erf}(x)$ is the error function [8] and $\Delta \omega_{c}$ is given by expression (20).

When the monochromator linewidth is much narrower than the interval of spectral coherence (19), the normalized dispersion tends to unity:

$$
\sigma_{W}^{2} \simeq 1 \quad \text { at } \quad \Delta \omega_{m} \ll \Delta \omega_{c}
$$

When the monochromator linewidth is much larger than the interval of spectral coherence, the dispersion is inversly proportional to the monochromator linewidth:

$$
\sigma_{W}^{2} \simeq \frac{\Delta \omega_{c}}{\Delta \omega_{m}} \quad \text { at } \quad \Delta \omega_{c} \ll \Delta \omega_{m}
$$

The next practical problem is to find the probability density distribution of the radiation energy after the monochromator, $p(W)$. We have shown above that the transversely coherent fraction of the synchrotron radiation possesses all the features of completely chaotic polarized radiation. Using the considerations similar to those presented in ref. [6] (Chapter 6) we find that the distribution of the radiation energy after the monochromator is described rather well by the gamma probability density distribution:

$$
p(W)=\frac{M^{M}}{\Gamma(M)}\left(\frac{W}{\langle W\rangle}\right)^{M-1} \frac{1}{\langle W\rangle} \exp \left(-M \frac{W}{\langle W\rangle}\right)
$$

where $\Gamma(M)$ is the gamma function of argument $M$ and

$$
M=\frac{1}{\sigma_{W}^{2}} .
$$

This distribution provides correct values for the mean value of $W$ and for the dispersion $\sigma_{W}^{2}$ :

$$
\int_{0}^{\infty} W p(W) d W=\langle W\rangle, \quad \int_{0}^{\infty} \frac{(W-\langle W\rangle)^{2}}{\langle W\rangle^{2}} p(W) d W=\frac{1}{M} .
$$

It follows from eq. (28) that parameter $M$ can not be less than unity. When parameter $M$ tends to unity, distribution (30) tends to the negative exponential distribution (12). When $M \gg 1$, distribution (30) tends to the Gaussian distribution. 


\section{Photoelectric detection of synchrotron radiation}

In this section we study the problem of photocount fluctuations in the detector of the SR. We assume that the radiation reaching the photodetector has full transverse coherence and that statistical properties of the radiation follow the laws described in the previous section. It has been shown above that the energy, $W$, in the radiation pulse is unpredictable. Thus, we can predict the probability density $p(W)$ only. In this case the probability of detection of $K$ photons is given by Mandel's semiclassical formula [6]:

$$
P(K)=\int_{0}^{\infty} \frac{(\alpha W)^{K}}{K !} \exp (-\alpha W) P(W) d W
$$

where $\alpha=\eta / \hbar \omega_{0}$ and $\eta$ is the quantum efficiency of the photodetector. Using formula (32) we get the expression for the mean and for the variance of the value of $K$ (see, e.g. ref. [6]):

$$
\langle K\rangle=\alpha\langle W\rangle, \quad \sigma_{K}^{2}=\frac{\left\langle K^{2}\right\rangle-\langle K\rangle^{2}}{\langle K\rangle^{2}}=\frac{1}{\langle K\rangle}+\sigma_{W}^{2},
$$

where $\sigma_{W}^{2}=1 / M$ is given by expression (28). The expression for photocounts fluctuations contains two terms. The first term corresponds to the "photon shot noise" and its origin is in the Poisson distribution. The second term corresponds to the classical fluctuations of the energy in the radiation bunch and takes its origin from the shot noise in the electron bunch. The ratio of the classical variance to the "photon shot noise" variance is named as the photocount degeneracy parameter $\delta_{c}[6]$ :

$$
\delta_{c}=\frac{\langle K\rangle}{M} .
$$

Let us consider a specific scheme with the monochromator installed in front of the photodetector. When monochromator linewidth is large, $\Delta \omega_{m} \gg \Delta \omega_{c}$, parameter $M$ is equal to the number of coherence intervals inside the monochromator linewidth and parameter $\delta_{c}$ can be interpreted as average number of photons detected within the coherence interval $\Delta \omega_{c}$ :

$$
\delta_{c} \simeq \frac{\Delta \omega_{c}}{\Delta \omega_{m}}\langle K\rangle \quad \text { at } \quad \Delta \omega_{m} \gg \Delta \omega_{c}
$$

In the opposite case, at $\Delta \omega_{m} \ll \Delta \omega_{c}$, the value of the parameter $M$ is close to the unity and parameter $\delta_{c}$ is equal to the average number of detected photons:

$$
\delta_{c} \simeq\langle K\rangle \quad \text { at } \quad \Delta \omega_{m} \ll \Delta \omega_{c}
$$


The probability density of the SR energy after the monochromator, $p(W)$, is the gamma distribution. Substituting (30) into Mandel's formula (32) and performing integration we come to the negative binomial distribution [6]:

$$
P(K)=\frac{\Gamma(K+M)}{\Gamma(K+1) \Gamma(M)}\left(1+\frac{M}{\langle K\rangle}\right)^{-K}\left(1+\frac{\langle K\rangle}{M}\right)^{-M}
$$

When monochromator has narrow linewidth, $\Delta \omega_{m} \ll \Delta \omega_{c}$, parameter $M$ tends to the unity, and the negative binomial distribution transforms to Bose distribution:

$$
\lim _{M \rightarrow 1} P(K)=\frac{\langle K\rangle^{K}}{(1+\langle K\rangle)^{K+1}}
$$

The negative binomial distribution tends to the gamma distribution at large values of the count degeneracy parameter $\delta_{c}$. In particular, the Bose distribution tends to the negative exponential distribution $\left(\delta_{c} \simeq\langle K\rangle\right.$ in this case):

$$
\lim _{\langle K\rangle \rightarrow \infty} \frac{\langle K\rangle^{K}}{(1+\langle K\rangle)^{K+1}}=\frac{1}{\langle K\rangle} \exp \left(-\frac{K}{\langle K\rangle}\right) .
$$

In the opposite case, at $\delta_{c} \rightarrow 0$, the negative binomial distribution (35) transforms to the Poisson distribution:

$$
\lim _{\delta_{c} \rightarrow 0} P(K)=\frac{\langle K\rangle^{K}}{K !} \exp (-\langle K\rangle)
$$

\section{The degeneracy parameter for synchrotron radiation}

The quality of the radiation source is described usually by the spectral brightness defined as the density of photons in the six-dimensional phase space volume (see, e.g. ref. [7]):

$$
B=\frac{1}{4 \pi^{2} \sigma_{z} \sigma_{z^{\prime}} \sigma_{x} \sigma_{x^{\prime}}}\left(\lambda \frac{d \dot{N}_{p h}}{d \lambda}\right)=\frac{1}{\epsilon_{x} \epsilon_{z}}\left(\lambda \frac{d \dot{N}_{p h}}{d \lambda}\right)
$$

where $\epsilon_{x, z}$ is the horizontal and the vertical photon beam emittance respectively. When the electron beam emittance is much larger than the diffraction limited photon beam emittance

$$
\epsilon_{x, z} \gg \frac{\lambda}{2}
$$


the photon beam emittance is approximately equal to the electron beam emittance.

Synchrotron radiation which emerges from a bending magnet has an uniform angular distribution in the deflection plane and we must therefore replace the divergence $\sigma_{x^{\prime}}$ by the total angle $\Delta \psi$ accepted by the photon beam line

$$
B=\frac{1}{4 \pi^{2} \sigma_{z} \sigma_{z^{\prime}} \sigma_{x}}\left(\lambda \frac{d^{2} \dot{N}_{\mathrm{ph}}}{d \lambda d \psi}\right)
$$

The expression in brackets (...) and photon flux $\Delta \dot{N}_{\text {ph }}$ into a spectral interval $\Delta \lambda / \lambda$ and into a horizontal angle $\Delta \psi$ are related by

$$
\Delta \dot{N}_{\mathrm{ph}}=\left(\lambda \frac{d^{2} \dot{N}_{\mathrm{ph}}}{d \lambda d \psi}\right) \frac{\Delta \lambda}{\lambda} \Delta \psi
$$

In practical units, the photon flux per one mrad into unit spectral bandwidth is

$$
\left(\lambda \frac{d^{2} \dot{N}_{p h}}{d \lambda d \psi}\right)[\text { phot. } / \text { sec. } / \text { mrad. }]=2.46 \times 10^{16} I(\mathrm{~A}) \mathcal{E}(\mathrm{GeV}) \eta\left(\lambda / \lambda_{c}\right)
$$

Here $\mathcal{E}$ is the energy of the particles, $I$ is the beam current, $\eta\left(\lambda / \lambda_{c}\right)$ is the universal spectral function, $\lambda_{c}=4 \pi R /\left(3 \gamma^{3}\right)$ is critical wavelength, $R$ is the radius of bend. For the large arguments $\lambda / \lambda_{c} \gg 1$ we may apply an asymptotic approximation for the spectral function $\eta\left(\lambda / \lambda_{c}\right)$ and get

$$
\eta\left(\lambda / \lambda_{c}\right) \simeq 2^{2 / 3} \Gamma(2 / 3)\left(\frac{\lambda_{c}}{\lambda}\right)^{1 / 3}
$$

We close this discussion about SR spectral brightness with some comments on the condition that were used to obtain result (37). Photons of SR are emitted into a narrow angle

$$
\Delta \theta_{c} \simeq\left(\frac{c}{\omega R}\right)^{1 / 3} \ll 1
$$

Hence the maximum linear dimension of a coherence area of the SR source is given approximately by: ${ }^{1}$

$$
\Delta x_{c} \simeq \Delta z_{c} \simeq\left(\frac{c^{2} R}{\omega^{2}}\right)^{1 / 3} \gg \lambda
$$

\footnotetext{
${ }^{1}$ It should be noticed that this fact should be taken into account when selecting coherent fraction of synchrotron radiation (see section 2 and Fig. 1).
} 
Thus the SR source is partially coherent when

$$
\varepsilon_{z}=2 \pi \sigma_{z} \sigma_{z^{\prime}} \gg \frac{\lambda}{2}, \quad \sigma_{x, z} \gg\left(\frac{c^{2} R}{\omega^{2}}\right)^{1 / 3} .
$$

Under this condition in eq. (37) values $\sigma_{x, z}, \sigma_{z^{\prime}}$ characterize the electron bunch.

It should be noted that in the opposite case, when

$$
\sigma_{x, z} \ll\left(\frac{c^{2} R}{\omega^{2}}\right)^{1 / 3}, \quad \sigma_{z^{\prime}} \ll\left(\frac{c}{\omega R}\right)^{1 / 3},
$$

in eq. (37) we must replace the $\sigma_{x, z}, \sigma_{z^{\prime}}$ by the $\left(c^{2} R / \omega^{2}\right)^{1 / 3},(c /(\omega R))^{1 / 3}$ respectively.

For the further consideration we use the notion of the wave degeneracy parameter $\delta_{W}$ which is equal to the average number of transversely coherent photons radiated by the electron bunch inside the spectral interval of coherence $\Delta \omega_{c}$. Physically this parameter describes the average number of photons which can interfere, or, according to the quantum theory, the number of photons in one quantum state (one "mode").

Taking into account that the "emittance" of the diffractionally limited photon beam is equal to (see, e.g. ref. [7]):

$$
\min \left(\epsilon_{x, z}\right)=\min \left(2 \pi \sigma_{x, z} \sigma_{x^{\prime} z^{\prime}}\right)=\frac{\lambda}{2}
$$

we calculate average number of spatially coherent photons radiated within one pulse into the spectral interval of $\Delta \lambda / \lambda$ :

$$
\left(\Delta N_{\mathrm{ph}}\right)_{\mathrm{coh}}=\frac{1}{4 \pi^{2} \sigma_{z} \sigma_{z^{\prime}} \sigma_{x}} \frac{\lambda^{2}}{4} \frac{\Delta \lambda}{\lambda} \int_{-\infty}^{\infty} d t\left(\lambda \frac{d^{2} \dot{N}_{p h}(t)}{d \lambda d \psi}\right)=\frac{\lambda^{2}}{4} \frac{\Delta \lambda}{\lambda} \int_{-\infty}^{\infty} d t B(t)
$$

where $B(t)$ is the instantaneous spectral brightness of the synchrotron radiation. The value of $\Delta \lambda / \lambda$ is connected with the interval of the spectral coherence by the relation:

$$
\frac{\Delta \lambda}{\lambda}=\frac{\Delta \omega_{c}}{\omega_{0}}=\frac{1}{\omega_{0}} \int_{-\infty}^{\infty}\left|g_{1}(\Delta \omega)\right|^{2} d(\Delta \omega)
$$

Using eqs. (19), (17) and Parseval's theorem we obtain:

$$
\frac{\Delta \omega_{c}}{\omega_{0}}=\frac{1}{\omega_{0}} \int_{-\infty}^{\infty}|\bar{F}(\Delta \omega)|^{2} d(\Delta \omega)=\frac{\lambda}{c} \int_{-\infty}^{\infty} F^{2}(t) d t
$$


Using the fact that the instantaneous spectral brightness $B(t)$ and the instantaneous value of the beam current $\langle I(t)\rangle$ are related by

$$
B(t)=\operatorname{const}\langle I(t)\rangle
$$

we can equivalently write (see eq. (1))

$$
B(t)=\text { const }^{\prime} F(t)
$$

It is convenient to refer to the peak value of spectral brightness

$$
\max B(t)=\text { const }^{\prime} \max F(t)=B_{\text {peak }} .
$$

By convention, we represent the time integral of the instantaneous spectral brightness in the form

$$
\int_{-\infty}^{\infty} B(t) d t=B_{\text {peak }} / \max F(t)
$$

Here the normalization condition

$$
\int_{-\infty}^{\infty} F(t) d t=1
$$

is used. Note that the dependence of the factor

$$
A=\frac{1}{\max F(t)} \int_{-\infty}^{\infty} F^{2}(t) d t
$$

on the exact shape of the bunch is rather weak. The results are $A=1$ for the rectangular pulse-shape and $A=2^{-1 / 2}$ for the Gaussian pulse-shape. Thus $A \simeq 1$ is a reasonable approximation. Finally, the degeneracy parameter can be estimated simply as:

$$
\delta_{W}=\frac{B_{\mathrm{peak}} \lambda^{3}}{4 c}
$$

The formula for calculation of the photocount degeneracy parameter $\delta_{c}$ is given by:

$$
\delta_{c}=\eta R_{m} \frac{\Delta \omega_{m}}{M \Delta \omega_{c}} \delta_{W}
$$


where $R_{m}$ is the integral reflection coefficient of the monochromator mirrors and the dispersive element. When the monochromator linewidth is large, $\Delta \omega_{m} \gg \Delta \omega_{c}$, parameter $\delta_{c}$ is given by:

$$
\delta_{c}=\eta R_{m} \delta_{W}
$$

In opposite case, at $\Delta \omega_{m} \ll \Delta \omega_{c}$, the value of the parameter $\mathrm{M}$ is close to the unity and the parameter $\delta_{c}$ is equal to:

$$
\delta_{c}=\eta R_{m} \frac{\Delta \omega_{m}}{\Delta \omega_{c}} \delta_{W}
$$

Let us present specific numerical example for the case of the storage ring DORIS $\left(\mathcal{E}=5 \mathrm{GeV}, I_{\text {peak }}=100 \mathrm{~A}, R=12 \mathrm{~m}, \sigma_{x}=2 \mathrm{~mm}, \sigma_{z} \sigma_{z^{\prime}}=0.1 \mathrm{~mm} \mathrm{mrad}\right)$. The peak spectral brightness at the wavelength of $\lambda=50 \AA$ is equal to:

$$
B \simeq 10^{14} \text { phot. } /\left(\mathrm{sec} \times \operatorname{mrad}^{2} \times \mathrm{mm}^{2} \times 0.1 \% \text { bandw. }\right)
$$

Substituting this number into eq. (41) we obtain that the wave degeneracy parameter is very small, about of $\delta_{W} \simeq 10^{-5}$.

At the wavelength of $\lambda \simeq 5000 \AA$ the spectral brightness reduces down to the value of:

$$
B \simeq 2 \times 10^{13} \text { phot. } /\left(\mathrm{sec} \times \operatorname{mrad}^{2} \times \mathrm{mm}^{2} \times 0.1 \% \text { bandw. }\right),
$$

but the value of $\lambda^{3}$ increases by six orders of magnitude with respect to the wavelength of $50 \AA$. As the result, in the visible wavelength range the degeneracy parameter is about of $\delta_{W} \simeq 2$.

Therefore, this numerical example shows that quantum fluctuations in the photodetector can be suppressed significantly by an appropriate choice of the operating wavelength.

\section{The expected value of the count-fluctuation product and its relation- ship to electron bunch formfactor}

In section 2 we described the layout of the device for measurement of the beam longitudinal profile. The detector of the synchrotron radiation is placed after the monochromator and is composed of a large number of discrete elements arranged in 
a closely packed linear array. Each element of the detector is connected with separate electronic counter. Let us consider two elements of the detector detecting the radiation in two different frequency intervals $\left(\omega_{1}, \omega_{1}+\Delta \omega_{1}\right)$ and $\left(\omega_{2}, \omega_{2}+\Delta \omega_{2}\right)$. The difference of frequencies $\left(\omega_{1}-\omega_{2}\right)$ is determined by the distance between the elements. The resolution of the monochromator is assumed to be better than $\Delta \omega_{1,2}$. During one radiation pulse, each of two elements of the detector registers $K_{1}$ and $K_{2}$ number of photocounts, respectively. After each shot, an electronic scheme multiplies these numbers and passes this product to an averaging accumulator, where it is added to the previously stored sum of count products. Finally, the total sum is divided by the number of shots. This result, averaged (shot to shot) count product $\left\langle K_{2} K_{2}\right\rangle$, contains information about the electron bunch formfactor.

In this section we perform a statistical analysis of the correlation between the counts of two elements of the detector. Let us calculate the expected average value of $\left\langle K_{1} K_{2}\right\rangle$ :

$$
\left\langle K_{1} K_{2}\right\rangle=\sum_{K_{1}=0}^{\infty} \sum_{K_{2}=0}^{\infty} K_{1} K_{2} P\left(K_{1}, K_{2}\right)
$$

where $P\left(K_{1}, K_{2}\right)$ is the joint probability distribution of $K_{1}$ and $K_{2}$. It follows from the basic properties of the conditional probabilities that

$$
P\left(K_{1}, K_{2}\right)=\int_{0}^{\infty} d W_{1} \int_{0}^{\infty} d W_{2} P\left(K_{1}, K_{2} \mid W_{1}, W_{2}\right) P\left(W_{1}, W_{2}\right)
$$

where $P\left(W_{1}, W_{2}\right)$ represents the joint probability distribution of energies $W_{1}$ and $W_{2}$. Since $K_{1}$ and $K_{2}$ are independent when conditioned by the $W_{1}$ and $W_{2}$, respectively, we can write $[6]$ :

$$
\begin{aligned}
& P\left(K_{1}, K_{2} \mid W_{1}, W_{2}\right)=P\left(K_{1} \mid W_{1}\right) P\left(K_{2} \mid W_{2}\right)= \\
& \frac{\left(\alpha W_{1}\right)^{K_{1}}}{K_{1} !} \exp \left(-\alpha W_{1}\right) \frac{\left(\alpha W_{2}\right)^{K_{2}}}{K_{2} !} \exp \left(-\alpha W_{2}\right) .
\end{aligned}
$$

Using eqs. (44), (45) and (46) one can show that the average value of the count product $\left\langle K_{1} K_{2}\right\rangle$ can be expressed in terms of the average of the classical energy product at the two detector elements:

$$
\left\langle K_{1} K_{2}\right\rangle=\alpha^{2}\left\langle W_{1} W_{2}\right\rangle
$$

Assuming the size of the detector element to be sufficiently small, $\Delta \omega_{1,2} \ll \Delta \omega_{c}$, the classical energies $W_{1}$ and $W_{2}$ can be written in the form:

$$
W_{1}=C_{1}\left|\bar{E}\left(\omega_{1}\right)\right|^{2} \Delta \omega_{1}, \quad W_{2}=C_{2}\left|\bar{E}\left(\omega_{2}\right)\right|^{2} \Delta \omega_{2}
$$


This allows one to express the average of the energy product $\left\langle W_{1} W_{2}\right\rangle$ in the terms of the second order spectral correlation function:

$$
\frac{\left\langle W_{1} W_{2}\right\rangle}{\left\langle W_{1}\right\rangle\left\langle W_{2}\right\rangle}=\frac{\left\langle\left|\bar{E}\left(\omega_{1}\right)\right|^{2}\left|\bar{E}\left(\omega_{2}\right)\right|^{2}\right\rangle}{\left\langle\left|\bar{E}\left(\omega_{1}\right)\right|^{2}\right\rangle\left\langle\left|\bar{E}\left(\omega_{2}\right)\right|^{2}\right\rangle} .
$$

Remembering that the SR source is a completely chaotic polarized radiation source, we can simplify expression (49) in the following way. Using eqs. (17) and (24) we obtain that the output signal of the counting interferometer is equal to the square of the modulus of the electron bunch formfactor:

$$
\frac{\left\langle\left(K_{1}-\left\langle K_{1}\right\rangle\right)\left(K_{2}-\left\langle K_{2}\right\rangle\right)\right\rangle}{\left\langle K_{1}\right\rangle\left\langle K_{2}\right\rangle}=\left|\bar{F}\left(\omega_{1}-\omega_{2}\right)\right|^{2} .
$$

One can see that the proposed device provides the possibility to measure the modulus of the Fourier transform of the electron bunch profile, while information about its phase is missing. To provide reconstruction of the beam profile from these data a special technique should be used similar to that developed by Hanbury-Brown and Twiss to determine a diameter of stars using the intensity interferometer [6].

\section{The signal-to-noise ratio associated with the output of the counting interferometer}

The next problem is to define the sensitivity of the proposed method for measurement of the electron bunch profile. The signal-to noise ratio for the proposed device can be written in the form: ${ }^{2}$

$$
\frac{S}{N}=\frac{\sqrt{N_{b}}\left\langle\Delta K_{1} \Delta K_{2}\right\rangle}{\sqrt{\left\langle\left(\Delta K_{1} \Delta K_{2}\right)^{2}\right\rangle-\left\langle\Delta K_{1} \Delta K_{2}\right\rangle^{2}}},
$$

where $\Delta K_{1,2}=\left(K_{1,2}-\left\langle K_{1,2}\right\rangle\right), N_{b}$ is the number of independent measurements averaged in the accumulator (total number of the electron bunches).

Let us consider the case when the degeneracy parameter is much larger than unity, $\delta_{c} \gg 1$. The fluctuations of the photocounts are defined mainly by the classical noise

\footnotetext{
${ }^{2}$ Complete analysis of the finite averaging should include the uncertainties associated with all of three average quantities: $\left\langle K_{1}\right\rangle,\left\langle K_{2}\right\rangle$ and $\left\langle\Delta K_{1} \Delta K_{2}\right\rangle$. For the purpose of simplicity we neglect the uncertainties of $\left\langle K_{1}\right\rangle$ and $\left\langle K_{2}\right\rangle$ (see, e.g. ref. [6]).
} 
in this case and eq. (51) can be reduced to

$$
\frac{S}{N}=\frac{\sqrt{N_{b}}\left\langle\Delta W_{1} \Delta W_{2}\right\rangle}{\sqrt{\left\langle\left(\Delta W_{1} \Delta W_{2}\right)^{2}\right\rangle-\left\langle\Delta W_{1} \Delta W_{2}\right\rangle^{2}}} .
$$

Remembering that the spectral density of the radiation energy is distributed in accordance with the negative exponential distribution (12) and using eq. (48) one can derive that

$$
\begin{aligned}
& \frac{\left\langle W_{1}^{2} W_{2}^{2}\right\rangle}{\left\langle W_{1}\right\rangle^{2}\left\langle W_{2}\right\rangle^{2}}=12\left(1+\left|\bar{F}\left(\omega_{1}-\omega_{2}\right)\right|^{4}\right), \\
& \frac{\left\langle W_{1}^{2} W_{2}\right\rangle}{\left\langle W_{1}\right\rangle^{2}\left\langle W_{2}\right\rangle}=2\left(1+2\left|\bar{F}\left(\omega_{1}-\omega_{2}\right)\right|^{2}\right), \\
& \frac{\left\langle W_{1,2}^{2}\right\rangle}{\left\langle W_{1,2}\right\rangle^{2}}=2 \\
& \frac{\left\langle W_{1} W_{2}\right\rangle-\left\langle W_{1}\right\rangle\left\langle W_{2}\right\rangle}{\left\langle W_{1}\right\rangle\left\langle W_{2}\right\rangle}=\left|\bar{F}\left(\omega_{1}-\omega_{2}\right)\right|^{2} .
\end{aligned}
$$

Combining the above expressions, we find that the signal-to-noise ratio is given by the expression:

$$
\frac{S}{N} \simeq \frac{\sqrt{N_{b}}\left|\bar{F}\left(\omega_{1}-\omega_{2}\right)\right|^{2}}{\sqrt{11\left|\bar{F}\left(\omega_{1}-\omega_{2}\right)\right|^{4}-12\left|\bar{F}\left(\omega_{1}-\omega_{2}\right)\right|^{2}+9}},
$$

at $\delta_{c} \gg 1$.

Let us perform analysis of the signal-to-noise ratio taking into account the quantum effects. In general case the joint probability $P\left(K_{1}, K_{2}\right)$ is given by eqs. (45) and (46). When calculating the signal component (numerator of eq. (51)), we can take into account classically induced fluctuations of the counts only. This can be done due to the fact that the quantum fluctuations of the counts at the outputs of two detectors are statistically independent. General calculations of the noise fluctuations associated with the output of counting interferometer (denominator of eq. (51)) should include both the classical and the quantum effects. Taking into account these considerations and using eq. (45) and (46), we can derive the following relations:

$$
\begin{aligned}
\frac{\left\langle K_{1}^{2} K_{2}^{2}\right\rangle}{\left\langle K_{1}\right\rangle^{2}\left\langle K_{2}\right\rangle^{2}} & =\frac{\left\langle\left(\alpha^{2} W_{1}^{2}+\alpha W_{1}\right)\left(\alpha^{2} W_{2}^{2}+\alpha W_{2}\right)\right\rangle}{\left\langle\alpha W_{1}\right\rangle^{2}\left\langle\alpha W_{2}\right\rangle^{2}} \\
\frac{\left\langle K_{1}^{2} K_{2}\right\rangle}{\left\langle K_{1}\right\rangle^{2}\left\langle K_{2}\right\rangle} & =\frac{\left\langle\left(\alpha^{2} W_{1}^{2}+\alpha W_{1}\right) \alpha W_{2}\right\rangle}{\left\langle\alpha W_{1}\right\rangle^{2}\left\langle\alpha W_{2}\right\rangle}
\end{aligned}
$$




$$
\frac{\left\langle K_{1,2}^{2}\right\rangle}{\left\langle K_{1,2}\right\rangle^{2}}=\frac{\left\langle\left(\alpha^{2} W_{1,2}^{2}+\alpha W_{1,2}\right)\right\rangle}{\left\langle\alpha W_{1,2}\right\rangle^{2}} .
$$

We illustrate the technique for calculations of the above expressions for the value of $\left\langle K_{1}^{2} K_{2}^{2}\right\rangle$ :

$$
\begin{aligned}
& \left\langle K_{1}^{2} K_{2}^{2}\right\rangle=\sum_{K_{1}=0}^{\infty} \sum_{K_{2}=0}^{\infty} K_{1}^{2} K_{2}^{2} P\left(K_{1}, K_{2}\right)= \\
& \int_{0}^{\infty} d W_{1} \int_{0}^{\infty} d W_{2} P\left(W_{1}, W_{2}\right) \times \\
& {\left[\sum_{K_{1}=0}^{\infty} K_{1}^{2} \frac{\left(\alpha W_{1}\right)^{K_{1}}}{K_{1} !} \exp \left(-\alpha W_{1}\right)\right]\left[\sum_{K_{2}=0}^{\infty} K_{2}^{2} \frac{\left(\alpha W_{2}\right)^{K_{2}}}{K_{2} !} \exp \left(-\alpha W_{2}\right)\right]=} \\
& \int_{0}^{\infty} d W_{1} \int_{0}^{\infty} d W_{2} P\left(W_{1}, W_{2}\right)\left(\alpha^{2} W_{1}^{2}+\alpha W_{1}\right)\left(\alpha^{2} W_{2}^{2}+\alpha W_{2}\right)= \\
& \left\langle\left(\alpha^{2} W_{1}^{2}+\alpha W_{1}\right)\left(\alpha^{2} W_{2}^{2}+\alpha W_{2}\right)\right\rangle .
\end{aligned}
$$

The expression for the signal-to-noise ratio can be calculated using eq. (54). This calculation is a lengthy one and we will consider only special case when the degeneracy parameter is much less than 1 . Using approximation $\delta_{c} \ll 1$ we obtain $\left(\delta_{c}\right)_{1,2} \simeq$ $\left\langle K_{1,2}\right\rangle$. Under such a condition it follows from eq. (54) that the variance $\left\langle\left(\Delta K_{1} \Delta K_{2}\right)^{2}\right\rangle$ is equal to:

$$
\left\langle\left(\Delta K_{1} \Delta K_{2}\right)^{2}\right\rangle \simeq\left\langle K_{1} K_{2}\right\rangle
$$

Thus, the signal-to-noise ratio is equal to:

$$
\frac{S}{N} \simeq \frac{\sqrt{N_{b}} \delta_{c}\left|\bar{F}\left(\omega_{1}-\omega_{2}\right)\right|^{2}}{\sqrt{1+\left|\bar{F}\left(\omega_{1}-\omega_{2}\right)\right|^{2}}} \quad \text { at } \quad \delta_{c} \ll 1 \text {. }
$$

Analysis of obtained asymptotics (53) and (56) for the signal-to-noise ratio allows one to make the following conclusions:

(i) The signal-to-noise ratio depends on the degeneracy parameter only when $\delta_{c}$ is much less than 1 . In this case the number of independent measurements at given signal-to-noise ratio is proportional to $1 / \delta_{c}^{2}$.

(ii) The signal-to-noise ratio is proportional to $|\bar{F}(\Delta \omega)|^{2}$. To hold the signal-tonoise ratio constant, the number of independent measurements must be proportional to $1 /|\bar{F}(\Delta \omega)|^{4}$. 


\section{Numerical example}

The operation of the new method for the electron pulse-shape measurement is illustrated for the TESLA Test Facility which is under construction at DESY [5]. It is supposed to use synchrotron radiation from the last bending magnet of the third bunch compressor (see Table 1). The value of the peak spectral brightness is equal to $10^{17}$ Phot. $/\left(\mathrm{sec} . \times \mathrm{mrad}^{2} \times \mathrm{mm}^{2} \times 0.1 \%\right.$ bandw.) and the degeneracy parameter is about of $\delta_{W} \simeq 10^{4}$ at the chosen operating wavelength of $\lambda=5000 \AA$.

We assume the use of a commercially available monochromator with the resolution of $\Delta \omega_{m} / \omega \simeq 3 \times 10^{-4}$. The electron bunch length is equal to $0.05 \mathrm{~mm}$ which corresponds to the relative value of the interval of spectral coherence of $\Delta \omega_{c} / \omega \simeq 3 \times 10^{-3}$. Comparing $\Delta \omega_{m}$ with $\Delta \omega_{c}$ one can conclude that ten pixels of the photodetector is sufficient to cover the interval of the spectral coherence. The photocount degeneracy parameter $\delta_{c}$ (see eq. (43)) will be of the order of $10^{2}$ at the quantum efficiency of the detector of $\eta \simeq 0.3$ and the value of the integral reflection coefficient of the monochromator mirrors and dispersive elements of $R_{m} \simeq 0.3$.

Table 1

Parameters of SR source

\begin{tabular}{ll}
\hline Electron beam & \\
Energy, $\mathcal{E}_{0}$ & $500 \mathrm{MeV}$ \\
Peak current, $I_{0}$ & $2.5 \mathrm{kA}$ \\
rms bunch length, $\sigma_{\mathrm{z}}$ & $50 \mu \mathrm{m}$ \\
Normalized rms emittance, $\epsilon_{\mathrm{n}}$ & $2 \pi \mathrm{mm} \mathrm{mrad}$ \\
Number of bunches per train & 7200 \\
Repetition rate & $10 \mathrm{~Hz}$ \\
Bending magnet & \\
\hline Length of magnet, $L_{\mathrm{w}}$ & $0.5 \mathrm{~m}$ \\
Magnetic field, $H$ & $1.3 \mathrm{~T}$ \\
$\beta$-function, & $11 \mathrm{~m}$ \\
Radiation & \\
Wavelength, $\lambda_{0}$ & $5000 \AA$ \\
Spectral coherence $\Delta \omega_{c} / \omega_{0}$ & $0.3 \%$ \\
Spectral brightness & $10^{17} \mathrm{Phot} . /\left(\mathrm{sec} . \times \mathrm{mrad}^{2} \times \mathrm{mm}^{2} \times 0.1 \%\right.$ bandw.) \\
Wave degeneracy parameter, $\delta_{W}$ & $10^{4}$ \\
\hline
\end{tabular}


The design value of the emittance of the electron bunch in the TTF accelerator is small, so the visible fraction of the SR from the magnet is always transversely coherent and there is no need in the installation of additional diaphragm (see Fig.1). If the monochromator is placed at the distance of $L=2 \mathrm{~m}$ from the radiation source, the aperture of the monochromator in the vertical direction should be not smaller than $\Delta \theta_{c} L \simeq 1 \mathrm{~cm}$. If vertical aperture is less than this value, the flux of coherent photons is decreased. The aperture of the monochromator in the horizontal plane can not be larger than $\Delta \theta_{c} L$ due to uniform distribution of the radiation in the horizontal plane.

Let us now estimate the number of shots required for achieving a given accuracy of the formfactor measurement. Suppose we wish to achieve a signal-to-noise ratio of 100 . The number of independent measurements required to achieve this accuracy depends on the value of the formfactor at given frequency. Using eq. (53) we find that the number of shots should be about $10^{5}$ at the value of the formfactor about of unity. If the linear array detector covers, for instance, the frequency range of $10 \Delta \omega_{c}$, one can perform ten independent measurements per one shot (per one radiation pulse). As a result one can decrease the number of shots which is required for a given accuracy by a factor of ten. In this case, one macropulse of the TTF accelerator should be sufficient to obtain given accuracy. This requires $10 \mathrm{MHz}$ data acquisition system. One can use photomultipliers or pin-photodiodes as photodetectors. In this case the light from the monochromator exit can be distributed to photomultipliers (or photodiodes) by means of optical fibers.

\section{Acknowledgement}

We wish to thank J. Feldhaus, P. Gürtler, G. Materlik, J.Pflüger, J. Roßbach, J. Schneider, S. Schreiber and B. Sonntag for useful discussions. 


\section{References}

[1] "Conceptual Design of a $500 \mathrm{GeV}$ e+e- Linear Collider with Integrated X-ray Laser Facility" (Editors R.Brinkmann, G. Materlik, J. Rossbach, A. Wagner), DESY 97-048, Hamburg, 1997.

[2] "Zeroth-Order Design Report for the Next Linear Collider", LBNL-PUB-5424,SLAC Report 474, UCRL-ID-124161 (May 1996).

[3] Status reports on CLIC, JLC and VLEPP are given in: Proceedings of the LC'92 ECFA Workshop on $e^{+} e^{-}$Linear Colliders (July,25-August,2,1993,Germany), MPI-PhE/9314, ECFA 93-154.

[4] R. Tatchyn et. al., Nucl. Instr. and Methods A375(1996)274.

[5] J. Rossbach, Nucl. Instr. and Methods A375(1996)269.

[6] J. Goodman, Statistical Optics, (John Willey and Sons, New York, 1985).

[7] H. Wiedemann, Particle Accelerator Physics II, (Springer-Verlag, Berlin-Heidelberg,1995).

[8] M. Abramowitz and I.A. Stegun, Handbook of Mathematical Function, (National Bureau of Standards, 1964). 